DePartment of ECONOMics ANd FInANCE Working PAPER SERIES • September 2004

\title{
Dynamic Programming: An Introduction by Example
}

\author{
Joachim Zietz * \\ Middle Tennessee State University, Murfreesboro, TN
}

\begin{abstract}
Some basic dynamic programming techniques are introduced by way of example with the help of the computer algebra system Maple. The emphasis is on building confidence and intuition for the solution of dynamic problems in economics. To better integrate the material, the same examples are used to introduce different techniques. One covers the optimal extraction of a natural resource, another consumer utility maximization, and the final example solves a simple real business cycle model. Every example is accompanied by Maple computer code to make replication and extension easy.
\end{abstract}

Key words: Dynamic Programming, Computer-Assisted Solutions, Learning by Example.

JEL category: C610, A230

${ }^{\dagger}$ A revised version is forthcoming in the Journal of Economic Education, Vol. 38, No. 2 (Spring 2007).

*Joachim Zietz, Professor, Department of Economics and Finance, Middle Tennessee State University, Murfreesboro, TN 37132, phone: 615-898-5619, email: jzietz@mtsu.edu., url: www.mtsu.edu/ jzietz. 


\section{Introduction}

The objective of this paper is to provide an introduction to dynamic programming that emphasizes intuition over mathematical rigor. ${ }^{1}$ The discussion is built around a set of examples that cover a number of key problems from economics. In contrast to other brief introductions to dynamic programming, such as King (2002), the paper integrates the use of a computer algebra system. ${ }^{2}$ Maple program code is provided for every example. ${ }^{3}$ Based on classroom experience, this allows readers not only to easily replicate and extend all problems but also to see more clearly their structure. The problems in this paper are fully accessible to undergraduates that are familiar with the Lagrangian multiplier method to solve contrained optimization problems.

Dynamic programming has strong similarities with optimal control, a competing approach to dynamic optimization. Dynamic programming has its roots in the work of Bellman (1957), while optimal control techniques rest on the work of the Russion mathematician Pontryagin and his coworkers in the late 1950s. ${ }^{4}$ While both dynamic programming and optimal control can be applied to discrete time and continuous time problems, most current applications in economics appear to favor dynamic programming for discrete time problems and optimal control for continous time problems. To keep the discussion reasonably simple, this paper will only deal with discrete time dynamic programming problems. These have become very popular in recent years in macroeconomics. However, the discussion will not be limited to macroeconomics but will try to convey the idea that dynamic programming has applications in other settings as well.

The paper is organized as follows. The next section provides some motivation for the use of dynamic programming techniques. This is followed by a discussion of finite horizon problems. Both numerical and non-numerical problems are treated. The subsequent section covers infinite horizon problems, again using both numerical and non-numerical examples. The last section before the concluding comments solves a simple stochastic infinite horizon problem of the real business cycle variety.

\section{A Motivating Example}

The basic principle of dynamic programming is best illustrated with an example. Consider for that purpose the problem of an oil company that wants to maximize profits from an oil well.

\footnotetext{
${ }^{1}$ Readers interested in more formal mathematical treatments are urged to read Sargent (1987), Stokey and Lucas (1989) or Ljungqvist and Sargent (2000).

${ }^{2}$ The book by Miranda and Fackler (2002) emphasizes numerical techniques to solve dynamic programming problems. The software package Matlab is used extensively. Adda and Cooper (2003) put less emphasis on computatioal aspects, but nonetheless make available a fair amount of computer code in both Matlab and Gauss.

${ }^{3}$ The brief overview of discrete time dynamic programming in Parlar (2000) was very helpful to the author because of its focus on coding in Maple.

${ }^{4}$ Kamien and Schwartz (1981) contains one of the most thorough treatments of optimal control. Most major textbooks in mathematical methods for economists also contain chapters on optimal control.
} 
Revenue at time $t$ is given as

$$
R_{t}=p_{t} u_{t}
$$

where $p$ is the price of oil and where $u$ is the amount of oil that is extracted and sold. In dynamic programming applications, $u$ is typically called a control variable. The company's cost function is quadratic in the amount of oil that is extracted,

$$
C_{t}=0.05 u_{t}^{2}
$$

The amount of oil remaining in the well follows the recursion or transition equation

$$
x_{t+1}=x_{t}-u_{t},
$$

where $x$ is known as a state variable in dynamic programming language. Since oil is a non-renewable resource, pumping out oil in the amount of $u$ at time $t$ means that there is exactly that much less left in the oil well at time $t+1$. We assume that the company applies the discount factor $\beta=0.9$ for profit streams that occur in the future. We also assume that the company intends to have the oil well depleted in 4 years, which means that $x_{4}=0$. This is known as a boundary condition in dynamic programming. Given these assumptions, the central question to be solved is how much oil should be pumped at time $t, t=0, \ldots, 3$ to maxime the discounted profit stream.

If one had never heard of dynamic programming or optimal control, the natural way to solve this problem is to set up a Lagrangian multiplier problem along the following lines,

$$
\max L(\mathbf{x}, \mathbf{u}, \boldsymbol{\lambda})=\sum_{t=0}^{3} \beta^{t}\left(R_{t}-C_{t}\right)+\sum_{t=1}^{4} \lambda_{t}\left(x_{t}-x_{t-1}+u_{t-1}\right),
$$

where $\mathbf{x}=\left(x_{1}, x_{2}, x_{3}\right)$, with $x_{0}$ given exogenously and $x_{4}=0$ by design, $\mathbf{u}=\left(u_{0}, u_{1}, u_{2}, u_{3}\right), \boldsymbol{\lambda}=$ $\left(\lambda_{1}, \lambda_{2}, \lambda_{3}, \lambda_{4},\right)$ and where the constraints simply specify that the amount of oil left at period $t$ is equal to the amount in the previous period minus the amount pumped in the previous period. Using standard techniques the above Lagrangian problem can be solved for the decision variables $\mathbf{x}$ and $\mathbf{u}$ and the Lagrangian multipliers $\boldsymbol{\lambda}$ in terms of the exogenous variables $\left\{p_{0}, p_{1}, p_{2}, p_{3}, x_{0}\right\}$. The Maple commands to solve this problem are given as follows:

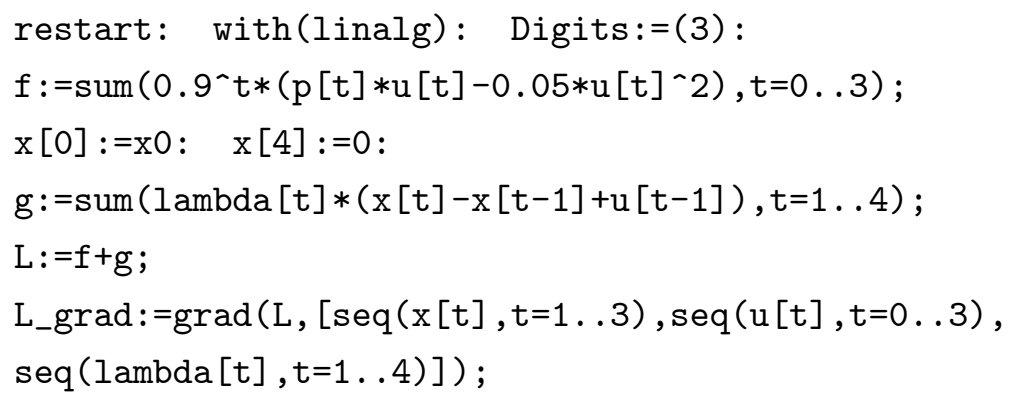


solve $\left(\left\{\operatorname{seq}\left(L \_\operatorname{grad}[i], i=1 \ldots 11\right)\right\},\{\operatorname{seq}(x[t], t=1 \ldots 3)\right.$,

$\operatorname{seq}(u[t], t=0.3)$, seq (lambda $[t], t=1 . .4)\})$;

$\operatorname{assign}(\%)$ :

For example, the optimal amounts of oil to pump in periods zero to four are given as

$$
\begin{aligned}
& u_{0}=.212 x_{0}+7.88 p_{0}-2.12 p_{1}-2.12 p_{2}-2.12 p_{3} \\
& u_{1}=.235 x_{0}-2.35 p_{0}+7.65 p_{1}-2.35 p_{2}-2.36 p_{3} \\
& u_{2}=.262 x_{0}-2.62 p_{0}-2.62 p_{1}+7.38 p_{2}-2.62 p_{3} \\
& u_{3}=.291 x_{0}-2.91 p_{0}-2.91 p_{1}-2.91 p_{2}+7.10 p_{3} .
\end{aligned}
$$

If one now specifies a particular sequence of prices and the value of $x_{0}$, then a complete numerical solution results. For example, assuming $\left\{x_{0}=1000, p_{0}=20, p_{1}=22, p_{2}=30, p_{3}=25\right\}$ the additional Maple commands

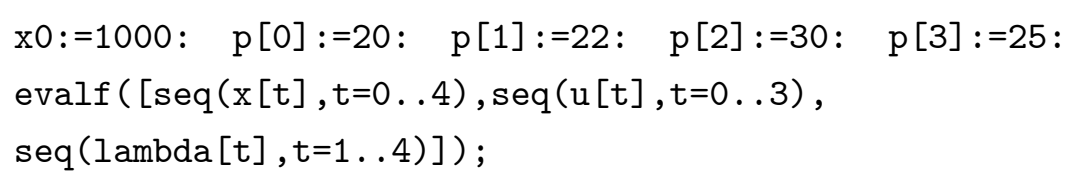

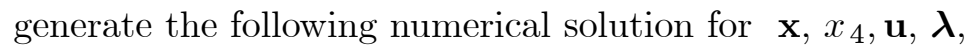

$[1000,794,566,260,0,206,227,308,260,0.60,0.60,0.60,0.60]$.

Given the relative simplicity with which the above problem can be solved with standard techniques, one may wonder where there is a room for a different approach. The clue to this puzzle lies in the simple fact that one cannot at time 0 pretend to know all future prices or actual amounts of oil that are pumped. For example, it may be that oil rigs break down or workers are on strike such that the optimal amount of oil simply cannot be pumped in a given year. Yet it should be clear that a change in any of the future $p$ or $x$ means that the above sequence of $x$ and $u$ is not optimal any longer. The whole problem needs to be recalculated subject to the new $p$ and the known values of $x$. Rather than recalculating the problem whenever something unexpected happens to either $p$ or $x$, one could try to find a decision rule or policy function for the control variable $u$ that can be followed regardless of random shocks to price or state variable $x$. That is the basic idea of dynamic programming. 


\section{Finite Horizon Problems}

\subsection{The Motivating Example Again}

We will now recalculate the above problem using dynamic programming techniques. The objective is, as before, to maximize

$$
\sum_{t=0}^{3} \beta^{t}\left(p_{t} u_{t}-0.05 u_{t}^{2}\right)
$$

subject to the transition equations

$$
x_{t+1}=x_{t}-u_{t} \quad t=0,1,2,3
$$

and the boundary condition

$$
x_{4}=0 \text {. }
$$

We assume again that the company applies the discount factor $\beta=0.9$.

The problem is solved with the help of the Bellman equations

$$
V_{t}=\max _{u_{t}}\left(p_{t} u_{t}-0.05 u_{t}^{2}\right)+0.9 V_{t+1}\left(x_{t}-u_{t}\right), \quad t=0,1,2,
$$

where $V_{t}$ is known as the value function in dynamic programming terminology. We note that the value function $V$ appears both on the left and on the right of the Bellman equation, although with a different time subscript. The solution process works backward. We start with the boundary condition and the value function for $t=3$ and then solve the problem recursively in the backward direction toward period 0 .

The starting point is the value function for $t=3$. Since $x_{4}=0$, the transition equation for period 3 is given as

$$
x_{4}=0=x_{3}-u_{3} .
$$

The transition equation for $t=3$ conveniently provides us with the optimal decision rule for the control variable $u$ in period 3 ,

$$
u_{3}=x_{3} .
$$

Equation 2 simply says to extract and sell all the oil that remains at the beginning of $t=3$ in that same period. Compared to the rule that we obtained from applying the Lagrange multiplier method in the previous section, equation 2 is much simpler because it is independent of any price variables or any other variables dated $t<3$. It is an application of the principle of optimality that 
underlies dynamic programming: we can do no better than just follow the derived optimal policy or decision rule regardless of what happens to control and state variables in earlier periods.

To obtain the optimal decision rules for the control variable in the periods prior to $t=3$, we will make use of the recursive nature of Bellman equation. One period at a time, we will move backward in time until we have found the decision rule for $t=0$.

The first step in finding the decision or policy rule for setting the control variable in $t=2$ consists of substituting the optimal policy function for $t=3$ into the value function for $t=3,^{5}$

$$
V_{3}=\left(p_{3} u_{3}-0.05 u_{3}^{2}\right)+0.9 V_{4}\left(x_{3}-u_{3}\right) .
$$

By equation 2, this simplifies to

$$
V_{3}=p_{3} x_{3}-0.05 x_{3}^{2}
$$

Next, we substitute equation 3 into the Bellman equation for period 2

$$
V_{2}=\max _{u_{2}}\left(p_{2} u_{2}-0.05 u_{2}^{2}\right)+0.9 V_{3}
$$

to obtain

$$
V_{2}=\max _{u_{2}}\left(p_{2} u_{2}-0.05 u_{2}^{2}\right)+0.9\left(p_{3} x_{3}-0.05 x_{3}^{2}\right) .
$$

Before we can maximize the right-hand side of equation 4 with respect to $u_{2}$ we need to consider that $x_{3}$ is connected with $u_{2}$ via the transition equation

$$
x_{3}=x_{2}-u_{2}
$$

Substituting equation 5 into 4 we obtain

$$
V_{2}=\max _{u_{2}}\left(p_{2} u_{2}-0.05 u_{2}^{2}\right)+0.9\left[p_{3}\left(x_{2}-u_{2}\right)-0.05\left(x_{2}-u_{2}\right)^{2}\right] .
$$

Now, only the decision variable $u_{2}$ and variables that are known or exogenous at time $t=2$ are in equation 6 . The right-hand side of equation 6 can be maximized with respect to the control variable $u_{2}$,

$$
\begin{gathered}
\frac{d\left[\left(p_{2} u_{2}-0.05 u_{2}^{2}\right)+0.9\left(p_{3}\left(x_{2}-u_{2}\right)-0.05\left(x_{2}-u_{2}\right)^{2}\right)\right]}{d u_{2}}=0 \\
p_{2}-0.19 u_{2}-.9 p_{3}+0.09 x_{2}=0 .
\end{gathered}
$$

\footnotetext{
${ }^{5}$ The value function is equal to the Bellman equation in (1), except that all occurrences of $u_{3}$ are replaced by the optimal value of $u_{3}$, which is $x_{3}$ according to equation 2 .
} 
Solving the above first-order condition for $u_{2}$ gives the optimal policy or decision rule for period 2 ,

$$
u_{2}=0.4737 x_{2}+5.263 p_{2}-4.737 p_{3}
$$

Similar to the decision rule in equation 2, this policy rule for the control variable is simpler than the correspondig rule from the Lagrangian multiplier approach in the last section. There is also no need to revise this rule in the light of unexpected shocks to price or the state variable $x$ prior to $t=2$. This completes the calculations for $t=2$.

To find the optimal policy rule for period 1, we need to insert equation 7 into the value function for period 2 to get an expression similar to equation 3. After some simplifications, the value function for period 2 can be written as

$$
V_{2}=.474\left(p_{2}+p_{3}\right) x_{2}+2.636 p_{2}^{2}-4.734 p_{2} p_{3}-0.02368 x_{2}^{2}+2.132 p_{3}^{2} .
$$

The value function for period 2 needs to be substituted into the Bellman equation for period 1

$$
V_{1}=\max _{u_{1}}\left(p_{1} u_{1}-0.05 u_{1}^{2}\right)+0.9 V_{2}
$$

One obtains

$$
\begin{aligned}
V_{1}= & \max _{u_{1}}\left(p_{1} u_{1}-0.05 u_{1}^{2}\right)+0.9\left(.474\left(p_{2}+p_{3}\right) x_{2}\right. \\
& \left.+2.636 p_{2}^{2}-4.734 p_{2} p_{3}-0.02368 x_{2}^{2}+2.132 p_{3}^{2}\right)
\end{aligned}
$$

Making use of the transition equation

$$
x_{2}=x_{1}-u_{1}
$$

in equation 8 , one can maximize its right-hand side with respect to $u_{1}$,

$$
\begin{aligned}
& d\left[\left(p_{1} u_{1}-0.05 u_{1}^{2}\right)+0.9\left(.474\left(p_{2}+p_{3}\right)\left(x_{1}-u_{1}\right)\right.\right. \\
& \frac{\left.\left.+2.636 p_{2}^{2}-4.734 p_{2} p_{3}-0.02368\left(x_{1}-u_{1}\right)^{2}+2.132 p_{3}^{2}\right)\right]}{d u_{1}}=0 .
\end{aligned}
$$

Solving the resulting first-order condition for $u_{1}$ one obtains

$$
u_{1}=.2989 x_{1}+7.013 p_{1}-2.989 p_{2}-2.989 p_{3} .
$$

This is the optimal policy or decision rule for period 1. We note that it has some similarities with the equation that determines the optimal value of $u_{1}$ from the Lagrangian multiplier approach. Yet, it does have fewer terms.

The last step in the solution of the optimal control problem is to obtain the decision or policy rule for period 0 . The procedure is the same as before. Insert the optimal decision rule for period 
1 into the Bellman equation for period 1 and simplify to obtain

$$
\begin{aligned}
V_{1}= & -.01494 x_{1}^{2}+3.006 p_{2}^{2}-2.989 p_{2} p_{3}+.2989\left(p_{1}+p_{2}+p_{3}\right) x_{1} \\
& +2.556 p_{3}^{2}+3.506 p_{1}^{2}-2.989 p_{1}\left(p_{2}+p_{3}\right)
\end{aligned}
$$

Next, the above value function is inserted into the Bellman equation for period 0 , which is given as

$$
V_{0}=\max _{u_{0}}\left(p_{0} u_{0}-0.05 u_{0}^{2}\right)+0.9 V_{1}
$$

After substituting out all occurrences of $x_{1}$ in the above equation with $\left(x_{0}-u_{0}\right)$, the right-hand side of equation 10 is maximized with respect to $u_{0}$. One obtains

$$
u_{0}=.2120 x+7.880 p_{0}-2.119 p_{1}-2.120 p_{2}-2.120 p_{3} .
$$

This decision rule for period 0 is the same as the one derived in the previous section using Lagrangian multiplier techniques.

The above results can be calculated by Maple with the help of the following code:

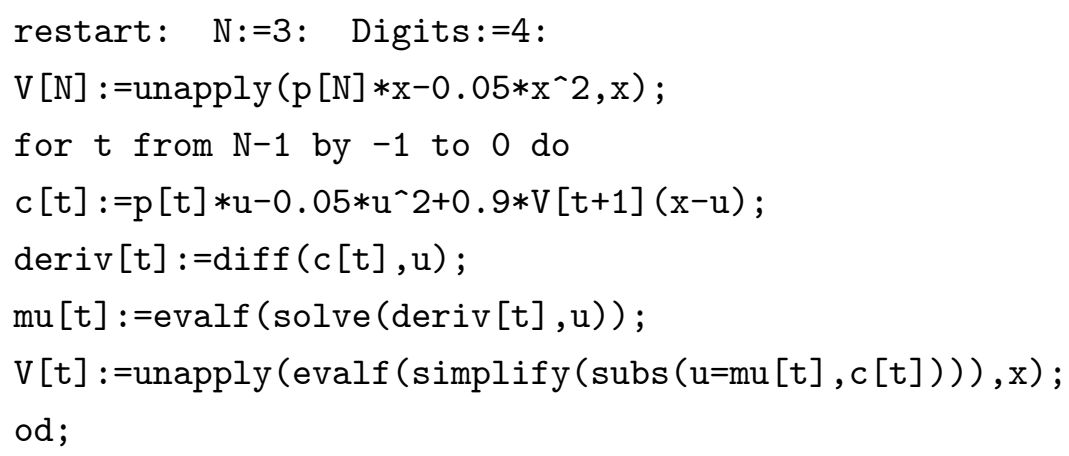

It should be apparent that the recursive structure of the dynamic programming problem makes it easy to extend the optimization to a larger number of periods.

\section{$3.2 \quad$ A Non-Numerical Example}

Much of the work in economics is about solving optimization problems without specific numerical entries. The next example applies the dynamic programming framework in this environment.

Consider the maximization of utility of a consumer that does not work but lives off a wealth endowment $(a)$. Assume that utility depends on the discounted logged value of consumption $(c)$ from period zero to two,

$$
\max U=\sum_{t=0}^{2} \beta^{t} \ln c_{t}
$$


where the discount factor is

$$
\beta=\frac{1}{1+r}
$$

and where $r$ is the relevant interest rate. Consumption is the model's control variable and the transition equations are given by

$$
a_{t+1}=(1+r) a_{t}-c_{t} \quad t=0,1,2
$$

The model's boundary condition is

$$
a_{3}=0
$$

which says that wealth is supposed to be depleted at the end of period 2. There are no bequests. The task is to find the optimal consumption sequence.

We start the solution process in the final period and work our way backward. Applying the boundary condition to the transition equation for period $t=2$ results in

$$
0=(1+r) a_{2}-c_{2} .
$$

This can be solved for $c_{2}$, the control variable for $t=2$, to get

$$
c_{2}=(1+r) a_{2}
$$

This is the decision rule for period $t=2$. We still need the decision rule for periods $t=1$ and $t=0$. To find these, we apply the Bellman equation

$$
V_{t}=\max _{c_{t}} \ln c_{t}+\beta V_{t+1}
$$

The Bellman equation for period 1 requires the value function of period 2. The latter is equal to the above Bellman equation for $t=2$, with $c_{2}$ replaced by its maximized value from equation 11 and with $V_{3}=0$ because wealth is exhausted at the end of $t=2$,

$$
V_{2}=\ln c_{2}=\ln \left[(1+r) a_{2}\right]
$$

This value function can now be inserted into the Bellman equation for period 1 ,

$$
V_{1}=\max _{c_{1}} \ln c_{1}+\beta V_{2}
$$

to obtain

$$
V_{1}=\max _{c_{1}} \ln c_{1}+\beta \ln \left[(1+r) a_{2}\right] .
$$


Before we can maximize the right-hand side of the Bellman equation with respect to $c_{1}, a_{2}$ has to be replaced using the transition equation for $t=1$,

$$
a_{2}=(1+r) a_{1}-c_{1}
$$

The Bellman equation for period 1 is now of the form,

$$
V_{1}=\max _{c_{1}} \ln c_{1}+\beta \ln \left[(1+r)\left[(1+r) a_{1}-c_{1}\right]\right]
$$

and its right-hand side can be maximized with respect to the control variable $c_{1}$. The first-order condition and $c_{1}$ are given as

$$
\begin{gathered}
\frac{d\left[\ln c_{1}+\beta \ln (1+r)+\beta \ln \left[a_{1}(1+r)-c_{1}\right]\right]}{d c_{1}}=\frac{-a_{1}(1+r)+c_{1}(1+\beta)}{c_{1}\left[-a_{1}(1+r)+c_{1}\right]}=0, \\
c_{1}=(1+r) \frac{a_{1}}{1+\beta} .
\end{gathered}
$$

The decision rule for $t=0$ still needs calculating. It requires the value function for $t=1$. This value function is equal to the Bellman equation for period 1, with $c_{1}$ replaced by the optimal decision rule, equation 12 ,

$$
V_{1}=\ln \frac{a_{1}(1+r)}{1+\beta}+\beta \ln \frac{a_{1}(1+r)^{2} \beta}{1+\beta} .
$$

Hence, the Bellman equation for $t=0$ is given as

$$
\begin{gathered}
V_{0}=\max _{c_{0}} \ln c_{0}+\beta V_{1} \\
V_{0}=\max _{c_{0}} \ln c_{0}+\beta \ln \frac{a_{1}(1+r)}{1+\beta}+\beta^{2} \ln \frac{a_{1}(1+r)^{2} \beta}{1+\beta}
\end{gathered}
$$

Substituting out $a_{1}$ with

$$
a_{1}=(1+r) a_{0}-c_{0}
$$

gives

$$
V_{0}=\max _{c_{0}} \ln c_{0}+\beta \ln \left((1+r) \frac{(1+r) a_{0}-c_{0}}{1+\beta}\right)+\beta^{2} \ln \left((1+r)^{2} \frac{\left((1+r) a_{0}-c_{0}\right) \beta}{1+\beta}\right)
$$

Maximizing the right-hand side with respect to the control variable $c_{0}$, results in the following 
first-order condition and optimal value for $c_{0}$

$$
\begin{gathered}
\frac{d V_{0}}{d c_{0}}=\frac{-a_{0}(1+r)+c_{0}\left(1+\beta+\beta^{2}\right)}{c_{0}\left(-a_{0}(1+r)+c_{0}\right)}=0 \\
c_{0}=(1+r) \frac{a_{0}}{1+\beta+\beta^{2}} .
\end{gathered}
$$

A comparison of the consumption decision rules for $t=0,1,2$ reveals a pattern,

$$
c_{t}=\frac{a_{t}(1+r)}{\sum_{i=0}^{2-t} \beta^{i}}, \quad t=0,1,2
$$

or, more generally, for $t=0, \ldots, n$

$$
c_{t}=\frac{a_{t}(1+r)}{\sum_{i=0}^{n-t} \beta^{i}}=\frac{a_{t}}{\beta \sum_{i=0}^{n-t} \beta^{i}}=\frac{a_{t}}{\sum_{i=1}^{n-t+1} \beta^{i}}, \quad t=0, \ldots, n .
$$

The ability to recognize and concisely describe such a pattern plays a key role in applications of dynamic programming in economics, in particular those applications that deal with infinite horizon problems. They will be discussed next.

The Maple program that solves the above consumption problem follows.

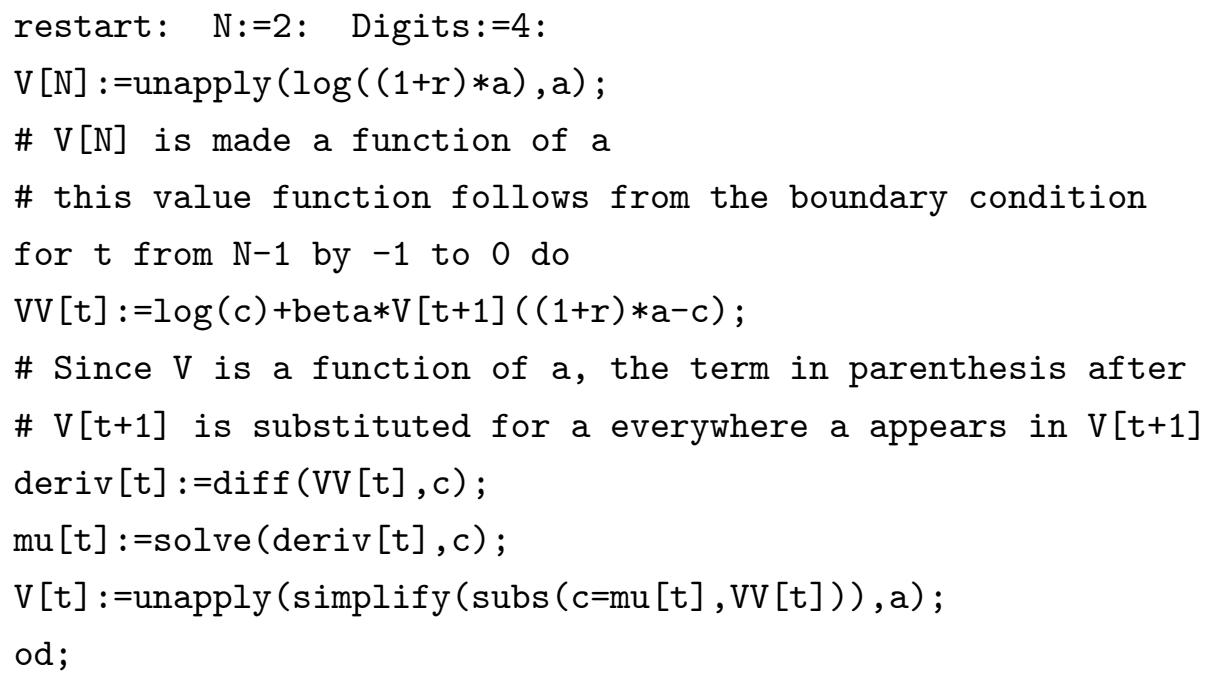

\section{Infinite Horizon Problems}

A question often posed in economics is to find the optimal decision rule if the planning horizon does not consist of a fixed number of $n$ periods but of an infinite number of periods. Examing the limiting case $n \rightarrow \infty$ may also be of interest if $n$ is not strictly infinite but merely large because taking the limit leads to a decision rule that is both simplified and the same for every period. Most 
often that is preferred when compared to a situation where one has to deal with a distinct and rather complex rule for each period from $t=0$ to $t=n$. We shall illustrate this point with the consumption problem of the last section.

\subsection{A Problem Without Numerics}

We want to modify the optimal consumption decision rule given in equation 14 so it applies for an infinite time horizon. For taking the limit, the first part of equation 14 is most useful,

$$
\lim _{n \rightarrow \infty} c_{t}=\lim _{n \rightarrow \infty} \frac{a_{t}(1+r)}{\sum_{i=0}^{n-t} \beta^{i}}
$$

In taking the limit, one needs to make use of the fact that

$$
\sum_{i=0}^{\infty} \beta^{i}=\frac{1}{1-\beta} .
$$

Application of this rule yields

$$
\lim _{n \rightarrow \infty} c_{t}=a_{t}(1+r)(1-\beta)=a_{t} r
$$

The decision rule for consumption in period $t$ simplifies to the intuitively obvious: consume in $t$ only what you get in interest from your endowment in $t$. The associated transition function for wealth is given as

$$
a_{t+1}=(1+r) a_{t}-c_{t}=(1+r) a_{t}-a_{t} r=a_{t},
$$

that is, wealth will remain constant over time.

This example has illustrated one way of arriving at optimal decision rules that apply to an infinite planning horizon: simply solve the problem for a finite number of periods, identify and write down the pattern for the evolution of the control variable, and take the limit $n \rightarrow \infty$. It should be obvious that the same procedure can be applied to identify the value function for time period $t$. To illustrate this point, recall the sequence of value functions for $t=1,2$

$$
\begin{gathered}
V_{2}=\ln \left[(1+r) a_{2}\right] \\
V_{1}=\ln \frac{a_{1}(1+r)}{1+\beta}+\beta \ln \frac{a_{1}(1+r)^{2} \beta}{1+\beta} .
\end{gathered}
$$


It is easy although tedious to verify that the value function for $t=0$ is given as

$$
V_{0}=\ln \frac{a_{0}(1+r)}{1+\beta+\beta^{2}}+\beta \ln \frac{a_{0}(1+r)^{2} \beta}{1+\beta+\beta^{2}}+\beta^{2} \ln \frac{a_{0}(1+r)^{3} \beta^{2}}{1+\beta+\beta^{2}} .
$$

One can proceed now as in the case of the consumption rule. The following pattern emerges from equations 15 to 17 ,

$$
V_{t}=\sum_{i=0}^{n-t} \beta^{i} \ln \frac{a_{t}(1+r)^{i+1} \beta^{i}}{\sum_{i=0}^{n-t} \beta^{i}}
$$

It is possible to check the correctness of this pattern by letting a program like Maple calculate the sequence of the $V_{t}$. For equations 15 through 17, the Maple code is given as

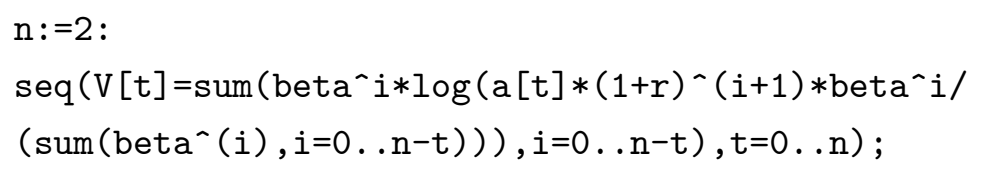

After one has verified that the formula is correct, one can take the limit $n \rightarrow \infty$

$$
\lim _{n \rightarrow \infty} V_{t}=\lim _{n \rightarrow \infty} \sum_{i=0}^{n-t} \beta^{i} \ln \frac{a_{t}(1+r)^{i+1} \beta^{i}}{\sum_{i=0}^{n-t} \beta^{i}} .
$$

Since interest centers on finding an expression involving the variable $a_{t}$, the idea is to isolate $a_{t}$. This can be done as follows

$$
\lim _{n \rightarrow \infty} V_{t}=\lim _{n \rightarrow \infty} \sum_{i=0}^{n-t} \beta^{i}\left(\ln a_{t}+\ln \frac{(1+r)^{i+1} \beta^{i}}{\sum_{i=0}^{n-t} \beta^{i}}\right)
$$

or

$$
\lim _{n \rightarrow \infty} V_{t}=\lim _{n \rightarrow \infty} \sum_{i=0}^{n-t} \beta^{i} \ln a_{t}+\lim _{n \rightarrow \infty} \sum_{i=0}^{n-t} \beta^{i} \ln \frac{(1+r)^{i+1} \beta^{i}}{\sum_{i=0}^{n-t} \beta^{i}} .
$$

The last expression can be rewitten as

$$
\lim _{n \rightarrow \infty} V_{t}=\lim _{n \rightarrow \infty} \sum_{i=0}^{n-t} \beta^{i} \ln a_{t}+\eta
$$

where $\eta$ is an expression independent of $a$. Taking the limit leads to

$$
\lim _{n \rightarrow \infty} V_{t}=\frac{1}{1-\beta} \ln a_{t}+\eta=\left(1+\frac{1}{r}\right) \ln a_{t}+\eta .
$$

With the help of a computer algebra program one could circumvent equation 18 and the following 
algebra altogether by going from equation 17 immediately to an expression like 19 for $n=2$. The sequence of Maple commands below takes equation 17, expands it, and isolates the terms in $a$.

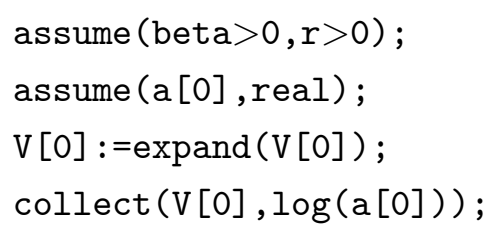

The output of these commands is

$$
V_{0}=\left(1+\beta+\beta^{2}\right) \ln a_{0}+\zeta
$$

where $\zeta$ consists of a number of terms in $\beta$ and $r$. One could do the same with equation 16 if one had trouble moving from equation 21 immediately to equations 19 and then 20.

\subsection{A Numerically Specified Problem}

Infinite horizon problems that are numerically specified can be solved by iterating on the value function. This will be demonstrated for the above consumption optimization problem. The task is to find the optimal decision rule for consumption regardless of time $t$. For that purpose we employ the Bellman equation without time subscripts,

$$
V(a)=\max _{c} \ln c+\beta V\left(a^{\prime}\right)
$$

where $a$ and $a^{\prime}$ denote current and future value of the state variable, respectively. The time subscripts are eliminated in the Bellman equation to reflect the idea that the value function $V(a)$ needs to satisfy the Bellman equation for any $a$. The iteration method starts with an initial guess of the solution $V(a)$, which we will identiy as $V^{(0)}(a)$. Guessing a possible solution is not as difficult as it sounds. Each class of problem typically has associated with it a general form of the solution. For example, in the current case we know from solving the consumption problem for $t=2$, that the value function will somehow depend on the $\log$ of $a$

$$
V^{(0)}(a)=A+B \ln a
$$

We will assume that $\beta=0.95$.

\subsubsection{A numerically specified initial guess}

We convert the initial guess of the value function into numerical form by assuming $A=B=1$,

$$
V^{(0)}(a)=\ln a .
$$


The initial guess $V^{(0)}(a)$ is substituted on the right-hand side of the Bellman equation

$$
V^{(1)}(a)=\max _{c} \ln c+0.95 \ln a^{\prime}
$$

Next, we make use of the tranition equation

$$
a^{\prime}=(1+0.05263) a-c
$$

to substitute out $a^{\prime}$

$$
V^{(1)}(a)=\max _{c} \ln c+0.95 \ln (1.05263 a-c)
$$

We maximize the right-hand side of $V^{(1)}(a)$ with respect to the control variable $c$,

$$
\frac{d V^{(1)}(a)}{d c}=\frac{1}{c}-\frac{0.95}{(1.05263 a-c)}=0 .
$$

which gives $c=0.53981 a$. Upon substituting the solution for $c$ back into $V^{(1)}(a)$ one obtains

$$
V^{(1)}(a)=\ln (0.53981 a)+0.95 \ln (1.05263 a-(0.53981 a))
$$

or after simplifying

$$
V^{(1)}(a)=-1.251+1.95 \ln a
$$

Since

$$
V^{(1)}(a) \neq V^{(0)}(a)
$$

we need to continue to iterate on the value function. For that purpose, we substitute the new guess, $V^{(1)}(a)$, into the Bellman equation and get

$$
V^{(2)}(a)=\max _{c} \ln c+0.95\left(-1.251+1.95 \ln a^{\prime}\right) .
$$

Substitution of the transition equation for $a^{\prime}$ gives

$$
V^{(2)}(a)=\max _{c} \ln c+0.95(-1.251+1.95 \ln (1.05263 a-c))
$$

which simplifies to

$$
V^{(2)}(a)=\max _{c} \ln c-1.1884+1.8525 \ln (1.05263 a-c)
$$


Maximizing the right-hand side of $V^{(2)}(a)$ with respect to $c$ one obtains $c=0.36902 a$. Substituting the optimal policy rule for $c$ back into $V^{(2)}(a)$ gives

$$
V^{(2)}(a)=\ln 0.36902 a-1.1884+1.8525 \ln (1.05263 a-0.36902 a)
$$

which simplifies to

$$
V^{(2)}(a)=-2.8899+2.8525 \ln a
$$

Since

$$
V^{(2)}(a) \neq V^{(1)}(a)
$$

another iteration is needed. Instead of continuing the above process of interating on the value function by hand, we make use of a Maple program to iterate until convergence is achieved,

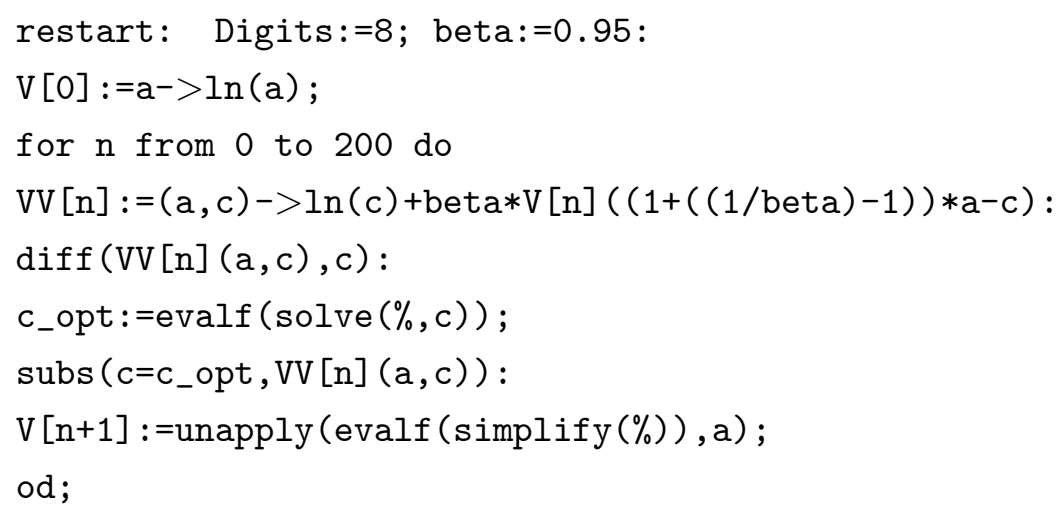

After about 200 iterations with eight digit accuracy, the value function reaches

$$
V(a)=19.9994 \ln a-58.886
$$

and the corresponding policy function for the control variable

$$
c=0.05263 a \text {. }
$$

These results are effectively identical to the ones that can be obtained from equation 20 and the corresponding decision rule for $c .{ }^{6}$ Specifically, equation 20 predicts the value function to be

$$
V(a)=20 \ln a+\eta
$$

\footnotetext{
${ }^{6}$ If the calculations are done with fewer digits accuracy, there is more of a discrepancy in results regardless of the number of iterations.
} 
whereas the corresponding decision rule for $c$ would be

$$
c=0.05263 a .
$$

\subsubsection{A numerically unspecified initial guess}

We start with the numerically unspecified guess

$$
V^{(0)}(a)=A+B \ln a .
$$

where $A$ and $B$ are constants to be determined. In this case, the value function will not be iterated on. This is of significant advantage if one cannot rely on the help of a computer algebra system. The solution process starts with the equation

$$
V(a)=\max _{c} \ln c+0.95 V^{(0)}\left(a^{\prime}\right)
$$

and the substitution of the initial guess

$$
V(a)=\max _{c} \ln c+0.95\left(A+B \ln a^{\prime}\right)
$$

Next, the transition equation is used to substitute out the state variable $a^{\prime}$

$$
V(a)=\max _{c} \ln c+0.95(A+B \ln (1.05263 a-c)) .
$$

The right-hand side of $V(a)$ is maximized with respect to the control variable $c$,

$$
\frac{d(\ln c+0.95(A+B \ln (1.05263 a-c)))}{d c}=0
$$

which results in

$$
c=\frac{21.0526 a}{20+19 B}
$$

The optimal $c$ is substituted back into $V(a)$, which, upon expansion, results in

$$
\begin{aligned}
V(a)= & 3.047+\ln a-\ln (20+19 B)+.95 A+2.846 B+.95 B \ln a \\
& +.95 B \ln B-.95 B \ln (20+19 B) .
\end{aligned}
$$

The steps up to this point have been standard. The solution process from here on is different.

If $V^{(0)}(a)$ had only one unknown parameter, say $A$, we could substitute $V^{(0)}(a)$ for $V(a)$ on the left side of the equation and then solve for the unknown value of $A$. With two unknown parameters, $A$ and $B$, to solve for, this method is not useful. An alternative solution procedure rests on the comparison of coefficients. In particular, one can compare the coefficient of $\ln a$ in $V(a)$ to $B$, 
the coefficient of $\ln a$ in $V^{(0)}(a)$, and, later, once $B$ is known, one can compare the value of the remainder of $V(a)$ to the parameter $A$ in $V^{(0)}(a)$.

To proceed with the coefficient comparison, we collect the terms in $\ln a$

$$
\begin{aligned}
V(a)= & 3.047+(1+0.95 B) \ln a-\ln (20+19 B)+.95 A+2.846 B \\
& +.95 B \ln B-.95 B \ln (20+19 B) .
\end{aligned}
$$

and set the coefficients of $\ln a$ in $V(a)$ and $V^{(0)}(a)$ equal to each other,

$$
(1+0.95 B)=B
$$

This results in $B=20$. To solve for the parameter $A$, we substitute $B=20$ into both $V(a)$ and $V^{(0)}(a)$, set the two equal, and solve for $A$,

$$
-2.9444+20.0 \ln a+.95 A=A+20 \ln a
$$

The solution is $A=-58.888$. With the parameters $A$ and $B$ known, the policy function for $c$ is given as

$$
c=0.05263 a
$$

and the value function is

$$
V(a)=-58.888+20.0 \ln a .
$$

Both match the ones obtained in earlier sections with other solution methods.

The Maple commands to replicate the above solution method are

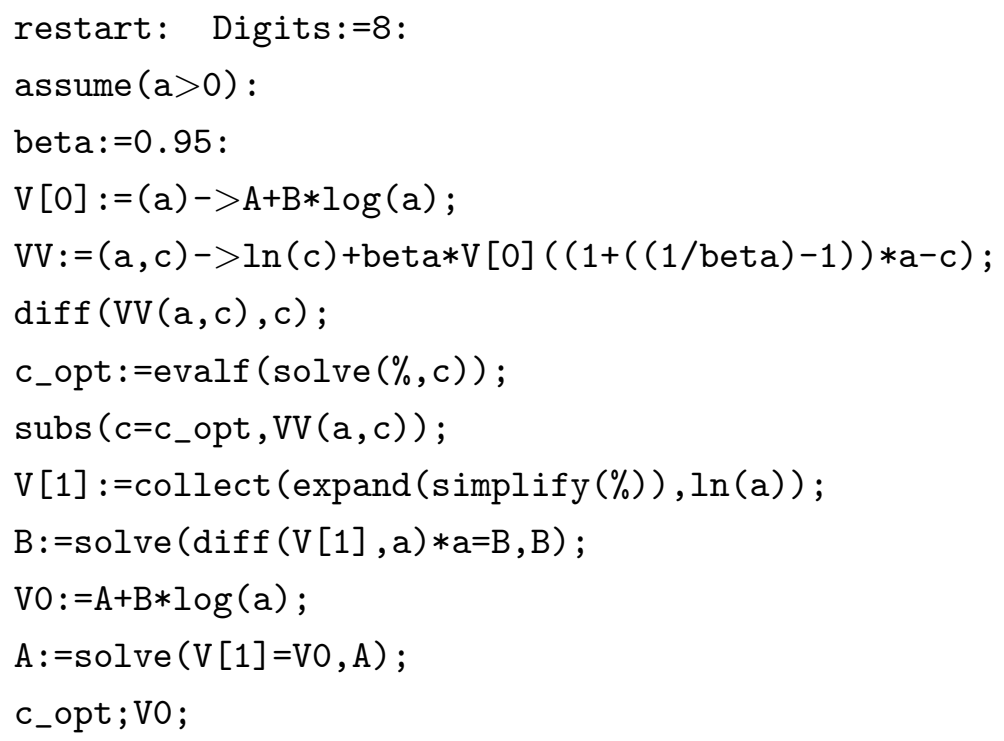




\section{A Stochastic Infinite Horizon Example}

Dynamic programming problems can be made stochastic by letting one or more state variables be influenced by a stochastic disturbance term. Most of the applications of dynamic programming in macroeconomics follow this path. One example is provided in this section.

We will consider the basic real business cycle (RBC) model discussed in King (2002), which is very much related to Kydland and Prescott (1982) and Long and Plosser (1983). Many RBC models are variations of this basic model.

The problem is to maximize the expected discounted sum of present and future utility, where utility is assumed to be a logarithmic function of consumption $(C)$ and leisure $(1-n)$

$$
\max E_{0} \sum_{y=0}^{\infty} \beta^{t}\left[\ln C_{t \square}+\delta \ln \left(1-\bar{n}_{t}\right)\right]
$$

where $n$ represents labor input and available time is set at unity. Maximization is subject to the market clearing constraint that output $(y)$ equals the sum of the two demand components, consumption and investment

$$
y_{t}=C_{t}+k_{t}
$$

where investment equals the capital stock $(k)$ because capital is assumed to depreciate fully in one period. There are two transition equations, one for each of the two state variables, output and the state of technology $(A)$,

$$
\begin{aligned}
y_{t+1} & =A_{t+1} k_{t}^{\alpha} n_{t}^{1-\alpha} \\
A_{t+1} & =A_{t}^{\rho} e^{\varepsilon_{t+1}}
\end{aligned}
$$

The transition equation of $y$ is a dynamic Cobb-Douglas production function and the transition equation of $A$ follows an autoregressive process subject to a disturbance term $(\varepsilon)$. $C$, $n$, and $k$ are the problem's control variables. To make the problem easier to follow, we use numbers for the parameters, $\beta=0.9, \alpha=0.3, \delta=1, \rho=0.8$.

The Bellman equation for this problem is given as

$$
V(y, A)=\max _{C, k, n} \ln C_{t}+\ln (1-n)+\beta E V^{(0)}(y, A),
$$

where $E$ is the expectations operator. The Bellman equation can be simplified by substituting for $C$ and, hence, reducing the problem to two control variables

$$
V(y, A)=\max _{k, n} \ln (y-k)+\ln (1-n)+0.9 E V^{(0)}(y, A)
$$

The next step in the solution process is to find an initial value function. In the earlier consumption 
problem, which was similar in terms of the objective function but had only one state variable $(a)$, we found that the value function was of the type

$$
V(a)=A+B \ln a
$$

Since there are two state variables in the present problem, we try the analogous solution

$$
V^{(0)}(y, A)=Z+G \ln y+H \ln A
$$

From here on, the problem follows the methodology used for the previous section. Substituting $V^{(0)}(y, A)$ into the Bellman equation gives

$$
V(y, A)=\max _{k, n} \ln (y-k)+\ln (1-n)+0.9 E(Z+G \ln y+H \ln A)
$$

Before the right-hand side of $V(y, A)$ can be maximized, $y$ and $A$ that appear in the expectations term are substituted out by their transition equations,

$$
V(y, A)=\max _{k, n} \ln (y-k)+\ln (1-n)+0.9 E\left(Z+G \ln \left(A^{0.8} e^{\varepsilon} k^{0.3} n^{0.7}\right)+H \ln \left(A^{0.8} e^{\varepsilon}\right)\right) .
$$

The right-hand side is expanded and then maximized with respect to both $k$ and $n$. This results in two first-order conditions that need to be solved simultaneously for the two control variables. The solution of the maximization process is given as

$$
k=\frac{0.27 G y}{1+0.27 G}, \quad n=\frac{0.63 G}{1+0.63 G} .
$$

These solutions are substituted back into the Bellman equation and the expectations operator is employed to remove the disturbance terms,

$$
\begin{aligned}
V(y, A)= & \ln \left(y-\frac{0.27 G y}{1+0.27 G}\right)+\ln \left(1-\frac{0.63 G}{1+0.63 G}\right) \\
& +0.9\left(Z+G\left(0.8 \ln A+0.3 \ln \frac{0.27 G y}{1+0.27 G}+0.7 \ln \frac{0.63 G}{1+0.63 G}\right)+H 0.8 \ln A\right)
\end{aligned}
$$

As in the consumption example, coefficients are compared between $V(y, A)$ and $V^{(0)}(y, A)$ to solve for the unknown values of $Z, G$, and $H$. To make a coefficient comparison feasible, we expand the right-hand side of $V(y, A)$, then simplify, and finally, collect the $\ln y$ and $\ln A$ terms to get

$$
V(y, A)=0.72(G+H) \ln A+(1+0.27 G) \ln y+\theta
$$


where

$$
\begin{aligned}
\theta= & 9.21-.9 G \ln G-.27 G \ln (100+27 G)-\ln (100+27 G) \\
& -\ln (100+63 G)+.9 Z-.63 G \ln (100+63 G)+3.5 G .
\end{aligned}
$$

Setting the coefficients of $\ln y$ in $V(y, A)$ and $V^{(0)}(y, A)$ equal to each other, we obtain the determining equation

$$
(1+0.27 G)=G
$$

which gives $G=1.37$. Similarly comparing the coefficients of $\ln A$ between $V(y, A)$ and $V^{(0)}(y, A)$,

$$
0.72(G+H)=H
$$

gives $H=3.52$. Making use of the solutions for $G$ and $H$ in $V(y, A)$, the value of $Z$ can be determined from the equation

$$
V(y, A)-1.37 \ln y-3.52 \ln A=Z
$$

as $Z=-20.853$.

With the values of $Z, G$, and $H$ known, the value function that solves the problem is given as

$$
V(y, A)=-20.853+1.37 \ln y+3.52 \ln A,
$$

while the policy functions or decision rules for $k, n$, and $C$ are

$$
k=0.27 y, \quad n=0.463 \quad C=0.73 y \text {. }
$$

We note that the addition of a stochastic term to the variable $A$ has no effect on the optimal decision rules for $k, n$, and $C$. This result is not an accident but, as discussed at the beginning of this paper, the key reason why dynamic programming is preferred over a traditional Lagrangian multiplier method in dynamic optimization problems: unpredictable shocks to the state variables do not change the optimal decision rules for the control variables. ${ }^{7}$

The above solution process can be automated and processed with other parameters if one uses the Maple commands below,

\footnotetext{
${ }^{7}$ The stochastic nature of state variables do affect how the state variables move through time. Different sequences of shocks to the state variables produce different time paths even with the same decision rules for the control variables. From a large number of such stochastic time paths, RBC researchers calculate standard deviations or other moments of the state and control variables and compare them to the moments of actually observed variables to check their models. Compare Adda and Cooper (2003) on this.
} 


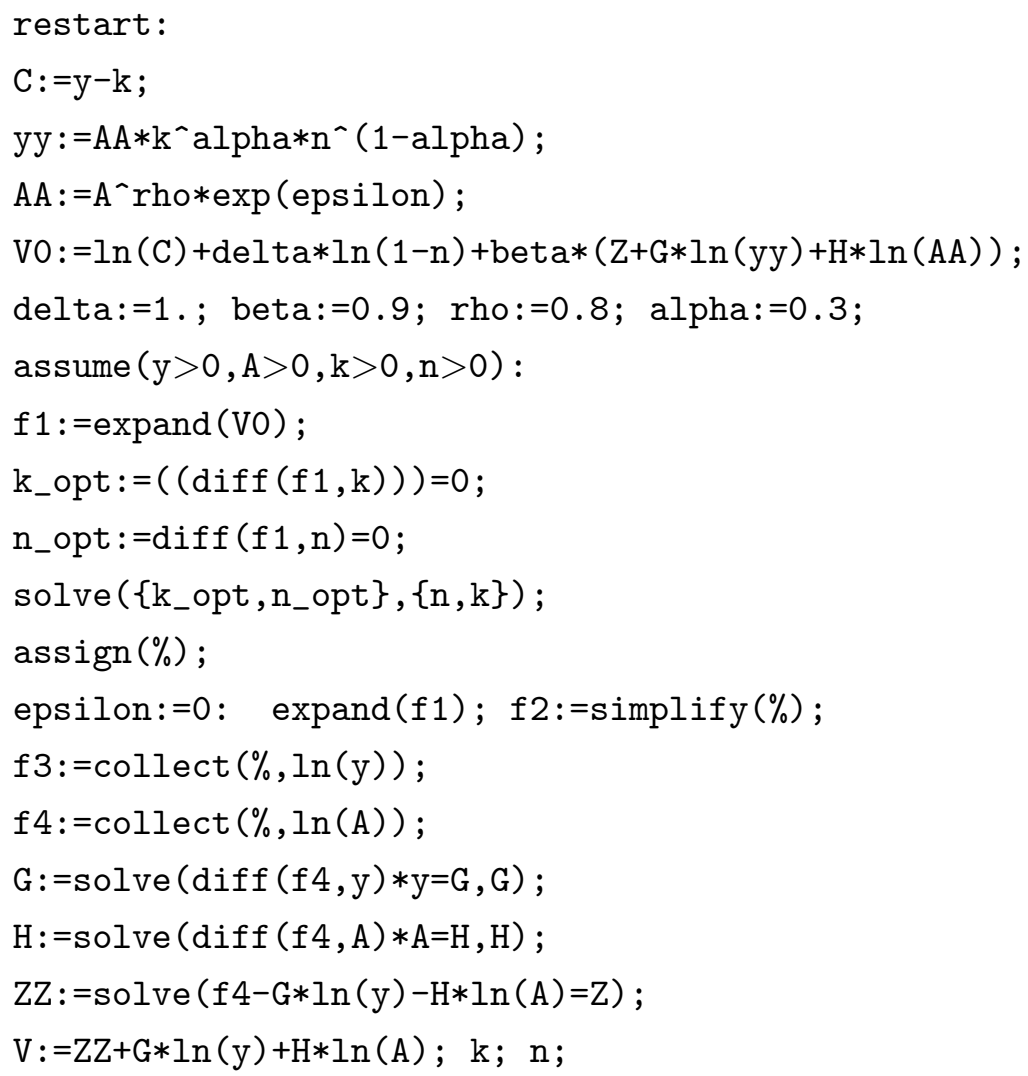

\section{Summary and Conclusion}

The purpose of this paper has been to introduce dynamic programming techniques by way of example with the help of the computer algebra system Maple. The emphasis of this introduction is to build confidence and intuition. The examples are solved one step at a time, with sufficient repetition of key steps to enhance the learning process. The coverage of solution techniques is limited to avoid confusion at the initial stages of learning. To better integrate the material, the same examples are used to introduce different techniques. Altogether, just three examples are employed, one on the optimal extraction of a natural resource, one on consumer utility maximization, and the final example covers a simple real business cycle model, which can be considered an extension of the utility maximization example.

Every example is accompanied by Maple computer code to make it possible for readers not only to replicate the given example but also to extend it in various directions and to encourage application of the techniques to other areas in economics. As the next step in the process of learning dynamic programming techniques, it is recommended to study and solve the economic examples discussed in Add and Cooper (2003). Some of them will seem immediately familiar based on the coverage in this paper. Others will open up new avenues of applying dynamic programming techniques. 


\section{References}

Add, J., and Cooper, R. (2003). Dynamic Economics: Quantitative Methods and Applications. MIT Press.

Kamien, M.I, and Schwartz, N.L. (1981). Dynamic Optimization: The Calculus of Variations and Optimal Control in Economics and Management, Elsevier North-Holland.

King, I.P. (2002). A Simple Introduction to Dynamic Programming in Macroeconomic Models. http://www.business.auckland.ac.nz/Departments/ econ/workingpapers/full/Text230.pdf.

Kydland, F., and Prescott, E. (1982). Time to Build and Aggregate Fluctuations. Econometrica 50, pp. 1345-1370.

Long, J., and Plosser, C. (1983). Real Business Cycles. Journal of Political Economy 90, pp. 39-69.

Ljungqvist, L. and Sargent, T.J. (2000). Recursive Macroeconomic Theory. MIT Press.

Miranda, M.J., and Fackler, P.L. (2002). Applied Computational Economics and Finance. MIT Press.

Parlar, M. (2000). Interactive Operations Research with Maple: Methods and Models. Birkhäuser. Sargent, T.J. (1987). Dynamic Macroeconomic Theory. Harvard University Press.

Stokey, N., Lucas, R.E., with Prescott, E. (1989). Recursive Methods for Economic Dynamics. Harvard University Press. 